\title{
UMA ANÁLISE DA COMPOSIÇÃO DE CUSTOS NO SEGMENTO DA ENGENHARIA CIVIL E SUAS PARTICULARIDADES
}

\author{
SANTOS, Victor Augusto Souza - victorr_augusto@hotmail.com \\ Graduado em Engenharia Civil e Pós-Graduando em MBA em Gestão Financeira, Auditoria \\ e Controladoria pela Faculdade do Sul da Bahia (FASB), Brasil. \\ Pós-Graduado em Docência do Ensino Superior pela Faculdade Luso Capixaba (FLC), \\ Brasil.
}

Professor Auxiliar I da Faculdade do Sul da Bahia (FASB) e Faculdade Pitágoras de

Teixeira de Freitas (FPTF), Brasil.

\section{RESUMO}

A área da engenharia civil na sua magnitude compreende desde a contratação da mão de obra e compra de materiais até a finalização da obra, seja ela edificação, infraestrutura e entre outros. Sendo assim, este artigo descreve de forma sucinta e coesa uma análise da composição de custos aplicado ao nicho da construção civil, vislumbrando-se a sua eficácia e significância. Para efetuar a gestão para com esta prestação de serviços, deve-se elaborar de maneira estruturada, um planejamento financeiro-econômico. É fundamental, ao trabalhar com a composição de custos, que o envolvido neste procedimento compreenda o quão relevante e imprescindível é este mecanismo no gerenciamento da organização. Pergunta-se: qual seria a métrica ideal para se confeccionar uma composição de custos no segmento da engenharia civil? Diante do exposto, este artigo tem como objetivo explicitar os procedimentos legais, gerenciais e fiscais relacionados a estas composições, além de demonstrar, através de planilhas e embasamentos teóricos e práticos, a metodologia de aplicação dos preceitos sobre as mesmas e suas respectivas incidências. Para esta explanação, será utilizada uma metodologia básica estratégica, explolatória, quantitativa e bibliográfica. Contudo, tem-se o intuito de alcançar um resultado que demonstre a eficiência da composição de custos realizada de forma correta, no qual desencadeie maior segurança para com a empresa e/ou profissional liberal, sendo coadjuvante na verificação da viabilidade de um empreendimento, em função de parâmetros técnicos fundamentados.

Palavras-chave: Constituição dos custos; Viabilidade; Planejamento financeiro. 


\title{
AN ANALYSIS OF THE COMPOSITION OF COSTS IN THE SEGMENT OF CIVIL ENGINEERING AND ITS PARTICULARITIES
}

\begin{abstract}
The area of civil engineering in its magnitude comprises from the hiring of labor and purchase of materials until the completion of the work, be it building, infrastructure and others. Thus, this article describes in a succinct and cohesive way an analysis of the composition of costs applied to the niche of the civil construction, glimpsing its effectiveness and significance. In order to manage this service delivery, a financial-economic planning should be elaborated in a structured manner. It is fundamental, when working with cost composition, that the one involved in this procedure understands how relevant and indispensable this mechanism is in managing the organization. Question: What would be the ideal metric to compose a cost composition in the civil engineering segment? In view of the above, this article aims to explain the legal, managerial and fiscal procedures related to these compositions, as well as to demonstrate, through spreadsheets and theoretical and practical bases, the methodology for applying the precepts about them and their respective incidences. For this explanation, a basic strategic, explitative, quantitative and bibliographic methodology will be used. However, it is intended to achieve a result that demonstrates the efficiency of the cost composition carried out in a correct way, in which it triggers greater security with the company and / or professional, being a supporting factor in the verification of the viability of a venture, in justified technical parameters.
\end{abstract}

Key words: Constitution of costs; Viability; Financial planning. 


\section{INTRODUÇÃO}

O setor da engenharia civil na sua magnitude envolve-se em diversas situações, desde a contratação da mão de obra e compra de materiais até a finalização da obra, seja ela em forma de residências, edifícios, condomínios, complexos industriais e etc. O ciclo produtivo desta área enfrenta diversos desafios, tanto favoráveis, quanto desfavoráveis. No caso das deficiências na gestão desses empreendimentos, especificadamente, sofrem por questões relacionadas a falta de planejamento financeiro. Percebe-se um conjunto de problemas nesse campo de atividade: insuficiência no detalhamento e na compatibilização de projetos, desqualificação da mão de obra e suas formas de contratação, planejamento tributário desestruturado, exigências e parâmetros oriundos de legislações ambíguas. Vê-se, então, o quão complexo é gerir este segmento. Em vista desse cenário, visualizam-se inúmeras recorrências de falhas provenientes dessas problemáticas, tais como: a não execução dos serviços com a devida qualidade, a subvalorização dos preços, o não cumprimento do prazo de entrega e entre outros fatores.

Em função disso, a atuação do engenheiro civil, correlacionado a vertente dos custos, é de suma importância, o mesmo é responsável pela elaboração e validação de orçamentos e planejamento de custos do empreendimento em todas as fases da obra e na gestão de custos e do fluxo de caixa, sendo que estes servem diretamente para o monitoramento e controle da evolução física da atividade empresarial.

O gerenciamento eficaz e eficiente torna uma empresa mais planejada e estruturada. Cuidar dos custos dentro de qualquer organização, é uma prioridade necessária na função de gerir, especificadamente, na área da construção civil.

É de grande valia ter ciência sobre os custos na construção civil, pois, desta forma torna-se este entendimento em ferramenta essencial para um controle dos serviços à serem prestados, no qual fundamenta-se todas as condições pertinentes à esta temática e respalda a empresa tecnicamente quanto ás condições de inviabilidades ou não. $\mathrm{O}$ monitoramento formal é um mecanismo imprescindível para tomada de decisões. Mediante tais considerações, alguns questionamentos emergem-se: Qual a importância da composição de custos no segmento da engenharia civil? Quais são os benefícios que estas composições podem trazer para uma empresa?

Neste tocante, este trabalho tem como intuito, analisar a composição de custos operacionais na área da engenharia civil. Para a consecução desse objetivo nuclear 
desenvolve-se dois objetivos específicos: a) explicitar os procedimentos legais, gerenciais e fiscais relacionados a composição de custos; b) demonstrar, mediante embasamento teórico e respectivos anexos, a metodologia de aplicação dos preceitos sobre composição de custos e suas incidências.

A metodologia atribuída tem por finalidade uma pesquisa básica e exploratória, pois busca-se suscitar o que é realizado comumente no mercado da engenharia civil. Procura-se, ainda, complementar algumas ferramentas que venham corroborar para com a melhoria da composição dos custos desse segmento em estudo.

Por termo, o resultado encontrado demonstra que a composição de custos realizada de forma correta, desencadeia segurança para a empresa, em relação a critérios de verificação de viabilidade de um empreendimento, tendo-se os respaldos técnicos embasados e fundamentados de maneira parametrizada.

\section{REFERENCIAL TEÓRICO}

\subsection{GERENCIAMENTO DOS CUSTOS DO PROJETO}

Conforme o PMI (2013), o gerenciamento de custo é composto por processos, como: planejar o gerenciamento de custos, estimar os custos, determinar o orçamento e controlar os custos, que tem por finalidade orientar e supervisionar para que o projeto seja realizado conforme orçamento aprovado. Os processos podem ser compreendidos por:

- Planejar o gerenciamento de custos - o processo de estabelecer as políticas, os procedimentos e a documentação para o planejamento, gestão, despesas e controle dos custos do projeto.

- Estimar os custos - o processo de estimar os recursos monetários necessários para concluir as atividades do projeto.

- Determinar o orçamento - o processo de agregação dos custos estimados de atividades individuais ou pacotes de trabalho para estabelecer uma linha de base dos custos autorizada.

- Controlar os custos - o processo de monitoramento do andamento do projeto para atualização do seu orçamento e gerenciamento das mudanças feitas na linha de base dos custos. 


\subsection{ESTIMATIVA DE CUSTO}

Conforme Mattos (2006), a composição de custos pode ser feita antes da execução do serviço, denominada estimativa de custo ou orçamento, e possui a função de oferecer uma aproximação do valor a ser gasto para a realização da atividade, sendo utilizado para definição de preços a serem atribuídos em propostas. Caso a elaboração da composição seja feita durante ou após a execução do serviço seu intuito passa a ser de controle de custos, auxiliando o responsável a identificar possíveis fontes de erros e construindo um histórico de referências para estimativas futuras.

Limmer (1997) e Dias (2011) afirmam que as composições de custos podem ser formadas por dois tipos diferentes de métodos, dependendo da qualidade da informação:

a) método da correlação: o custo é estimado através da correlação entre medidas de grandeza da construção e um valor atribuído a cada unidade desta medida. Há dois processos, o da correlação simples, em que produtos semelhantes têm cada um custo proporcional à sua dimensão característica, e o da correlação múltipla, no qual o custo do projeto é a soma dos custos obtidos da correlação e de suas diversas partes componentes;

b) método da quantificação: abrange dois processos distintos, o de quantificação de insumos e o de composições de custos unitários dos serviços. O método da quantificação de insumos levanta todos os insumos básicos necessários à execução da obra, que compõem os grupos de materiais, mão de obra e equipamentos. A composição de custos unitários de serviço é baseada nos serviços a serem executados, ou seja, o custo de cada serviço é obtido por meio da utilização de composições unitárias, que relacionam o consumo de materiais, mão de obra e equipamentos necessários à execução de uma unidade de serviço.

Dias (2011) mostra, através de uma sequência de processos, como alcançar com maior precisão a estimativa de custo através do método da quantificação, conforme a tabela a seguir: 
Tabela 1 - Acurácia de Elaboração de uma Estimativa de Custos

\begin{tabular}{|c|c|}
\hline Quantificação de Insumos & $\begin{array}{l}\text { Composições de Custos Unitários dos } \\
\text { Serviços }\end{array}$ \\
\hline - Levantamento de quantidades de insumos; & • Levantamento de quantidades; \\
\hline $\begin{array}{l}\text { - Pesquisa de mercado de preços de insumos: } \\
\text { mão de obra, materiais, equipamentos e } \\
\text { tributos; }\end{array}$ & $\begin{array}{l}\text { - Pesquisa de mercado de preços de insumos: } \\
\text { mão de obra, materiais, equipamentos e } \\
\text { tributos; }\end{array}$ \\
\hline $\begin{array}{l}\text { - Cálculo dos preços parciais por insumos, } \\
\text { segundo formulários próprios; }\end{array}$ & $\begin{array}{l}\text { - Definição das composições de custos } \\
\text { unitários dos serviços; }\end{array}$ \\
\hline $\begin{array}{l}\text { - Definição dos custos indiretos e cálculo do } \\
\text { BDI ou preenchimento de formulários } \\
\text { próprios que não representam o BDI; }\end{array}$ & $\begin{array}{l}\text { - Definição dos custos indiretos e cálculo do } \\
\text { BDI; }\end{array}$ \\
\hline - Cálculo do preço global de venda. & $\begin{array}{l}\text { - Cálculo dos preços unitários dos serviços; } \\
\text { - Cálculo do preco global de venda. }\end{array}$ \\
\hline
\end{tabular}

Fonte: Elaborado pelo autor, a partir de Dias (2011)

\subsection{VARIÁVEIS DE UMA ESTIMATIVA DE CUSTOS}

Limmer (1997) menciona que para a elaboração de uma composição de custos não se pode adotar valores fixos para os parâmetros e insumos a serem utilizados devido à complexidade, incertezas e dinâmica que ocorre no setor da construção civil, como, a inflação sobre os materiais, inconstância da produtividade da mão-de-obra, leis sociais e condições de trabalhos que variam de acordo com a localidade, entre outros.

Segundo Dias (2011) pode-se citar as seguintes variáveis de uma estimativa de custos:

- BDI - benefício e despesas indiretas;

- Encargos sociais;

- Tributos sobre o preço de venda;

- Composição de custos unitários;

“Todas as variáveis de um orçamento em uma construção deverão ser calculadas projeto por projeto, pois a obra é um serviço único (DIAS, 2011, p. 19).”

\subsubsection{BDI - Benefício e Despesas Indiretas}

O BDI, sigla que denomina Beneficio (ou Bonificação) e Despesas Indiretas, é o percentual relativo ao lucro e despesas indiretas que incidirá sobre os custos diretos. De acordo com Mattos (2006) o BDI é o fator a ser aplicado ao custo direto para obtenção do preço de venda. Sendo PV o preço de venda e CD o custo direto, a fórmula é: 


$$
\mathrm{PV}=\mathrm{CD} \times\left(1+\frac{\mathrm{BDI}}{100}\right)
$$

Mattos (2006) afirma que cada obra terá seu BDI, devido as diferenças que ocorrem em alguns parâmetros, como: a administração central e local, imprevistos e contingências, composição de custos, lucro e impostos. Conforme Dias (2008), o percentual do BDI tem que ser justificado pela análise das variáveis que o compõem, conforme a metodologia a seguir:

$$
\mathrm{BDI}=\left(\left(\frac{(1+\mathrm{AC}+\mathrm{CF}+\mathrm{S}+\mathrm{G}+\mathrm{MI})}{1-(\mathrm{TM}+\mathrm{TE}+\mathrm{TF}+\mathrm{MBC})}\right)-1\right) \times 100
$$

\begin{tabular}{|c|c|}
\hline $\begin{array}{c}\text { Variáveis incidentes sobre o } \\
\text { custo: }\end{array}$ & $\begin{array}{c}\text { Variáveis incidentes sobre o } \\
\text { preço de venda: }\end{array}$ \\
\hline $\begin{array}{l}\text { AC - Administração Central } \\
\text { CF - Custo Financeiro } \\
\text { S - Seguros } \\
\text { G - Garantia } \\
\text { MI - Margem de Incerteza ou de } \\
\text { erro (somente para contratantes) }\end{array}$ & $\begin{array}{l}\text { TM - Tributos Municipais } \\
\text { TE - Tributos Estaduais } \\
\text { TF - Tributos Federais } \\
\text { MBC - Margem Bruta de } \\
\text { Contribuição }\end{array}$ \\
\hline
\end{tabular}

Tabela 2 - Elementos do cálculo do BDI

Fonte: Elaborado pelo autor, a partir de Dias (2008)

\subsubsection{Encargos Sociais}

"Encargos sociais são os custos incidentes sobre a folha de pagamento de salários e têm sua origem na CLT - Consolidação das Leis do Trabalho, na Constituição Federal de 1988, em leis específicas e nas convenções coletivas de trabalho. (SINAPI, 2017, p. 66).”

Deve-se calcular a taxa de leis sociais conforme o tipo de contratação do profissional, podendo ser, por hora ou mês (DIAS, 2011).

a) Salários de mensalistas - nesse caso alguns itens, como, repouso semanal remunerado e os dias de feriados estão incorporados nos salários, ao contrário do salário hora que considera esses itens como encargos sociais. É adotado, no máximo, um total entre 170 horas de trabalho por mês.

b) Salários de horistas - não há encargos embutido no salário hora, eles são pagos aos empregados complementarmente. Por lei considera-se 220 horas de trabalho por mês.

c) Encargos sobre horas extras - são vários aspectos a adotar conforme o tipo de hora extra considerado, isto é, noturna, sábado, domingo, feriado, bem como, combinações entre estas e etc. Entretanto, para cálculo da hora extra divide-se o salário mês por 220 horas.

Para efeito de cálculo, os encargos são divididos em cinco grupos (SINAPI, 2017): 


\subsubsection{GRUPO A - Encargos Sociais Básicos}

Correspondem aos tributos estabelecidos por lei para serem pagos pelas empresas sobre os salários, que oferece benefícios aos empregados. São eles: INSS, SENAI, SESI, SEBRAE, INCRA, salario educação, seguros de acidentes sobre o trabalho.

\subsubsection{GRUPO B - Encargos Sociais que recebem a incidência do Grupo A}

Correspondem aos valores pagos ao empregado como salário referente aos dias em que não há prestação de serviços. Deve-se considerar, neste caso, $13^{\circ}$ Salário, Férias, Aviso Prévio, Abonos, entre outros.

\subsubsection{GRUPO C - Encargos Sociais que não recebem incidência do Grupo A e do Grupo B}

São predominantemente indenizatórios e correspondem ao aviso prévio, valores para rescisão sem justa causa e outras indenizações.

\subsubsection{GRUPO D - Reincidência entre os Grupos A e B}

Correspondem ao cálculo da incidência cumulativa do Grupo A sobre o Grupo B. O Grupo D é obtido através da multiplicação do percentual encontrado para o Grupo A pelo Grupo B e dividido por 100.

\subsubsection{GRUPO E - Encargos complementares}

Estes encargos obrigatórios são aqueles oriundos de Acordos Coletivos ou de Convenção Coletiva de Trabalho entre os sindicatos patronais e os laborais, das Legislações Federais, das Normas Regulamentadoras de Segurança e Saúde no Trabalho do Ministério do Trabalho e Emprego - NR 18 e NR 6 (EPI) e NR 7.

Assim, o percentual total dos encargos sociais pode ser calculado a partir da seguinte fórmula:

ENCARGOS SOCIAIS = GRUPO A + GRUPO B + GRUPO C + GRUPO D + GRUPO E

\subsubsection{Tributos Sobre o Preço de Venda}

O elevado valor da tributação no Brasil alerta para possíveis transtornos financeiros, caso não seja adequadamente considerada nas composições de custos (DIAS, 2008). Os tributos incidentes sobre cada componente da composição estão relacionados na tabela a seguir: 
Tabela 3 - Tributos Incidentes sobre a composição

\begin{tabular}{|l|l|}
\hline \multicolumn{1}{|c|}{ Insumos } & Tributos Incidentes \\
\hline Mão de Obra & Encargos Sociais \\
Materiais & IPI e ICMS \\
Equipamentos & IPI e ICMS \\
Tributos sobre a Receita & ISS, PIS e COFINS \\
Tributos sobre o Lucro & IRPJ e CSLL \\
\hline
\end{tabular}

De acordo com um estudo de caso, Dias (2008) apresenta a seguinte análise em relação ao percentual de tributos que atuam sobre os insumos de uma composição de custos:

Tabela 4 - Análise de Tributos Sobre a Construção

\begin{tabular}{|l|c|c|c|}
\hline \multicolumn{1}{|c|}{ Insumos } & $\begin{array}{c}\text { \% sobre o } \\
\text { Preço }\end{array}$ & $\begin{array}{c}\text { Carga } \\
\text { Tributária }\end{array}$ & \% sobre o Preço \\
\hline Mão de Obra & $39,00 \%$ & $54,50 \%$ & $21,30 \%$ \\
\hline Materiais & $40,60 \%$ & $24,00 \%$ & $9,70 \%$ \\
\hline Equipamentos & $4,00 \%$ & $25,00 \%$ & $1,00 \%$ \\
\hline Tributos sobre a Receita & $6,70 \%$ & $100,00 \%$ & $6,70 \%$ \\
\hline Lucro Bruto / Tributos sobre o Lucro & $9,70 \%$ & $25,00 \%$ & $2,40 \%$ \\
\hline Média dos Tributos sobre a Construção & $100,00 \%$ & $41,10 \%$ \\
\hline
\end{tabular}

\subsubsection{Composição Analítica de Custos Unitários}

Mattos (2006) assegura que a composição analítica de custos é a ferramenta adequada para chegar ao custo da obra de forma precisa e detalhada. É feito através das composições de custos e atribuições de preços de insumos.

De acordo com Dias (2011) devem ser considerados na composição analítica de custos as classes de: equipamentos, mão-de-obra, materiais, transportes, subempreiteiros e BDI. A metodologia de estimativa da composição está descrita através da sequência a seguir:

a) Equipamentos

Deve-se listar todos os equipamentos a serem utilizados na composição, com suas respectivas descrições, quantidades, produtividade e porcentagens de utilização, sendo incluso a mão-de-obra do operador.

b) Mão-de-obra

Todos os profissionais envolvidos diretamente no serviço devem ser constatados, assim como a sua respectiva quantidade de horas necessária para a produção. Os operadores de equipamentos e profissionais indiretos (engenheiros, mestre de obras e funcionários 
administrativos) não estão inclusos nessa categoria, sendo esse último inserido em Bonificações e Despesas Indiretas.

c) Materiais

É fundamental listar e especificar os serviços a serem executados, de maneira sucinta e clara, para uma melhor eficácia ao levantar o material a ser utilizado e suas devidas quantidades.

d) Transportes

Se o preço do transporte for considerado pequeno ou caso a natureza do transporte seja verificada como de uso geral, pode-se adicionar seu valor ao custo indireto. $\mathrm{O}$ valor referente ao transporte comercial dos materiais, devem estar inclusos no serviço.

e) Cálculo do custo unitário direto

Finalizando os processos anteriores, assim como a pesquisa de mercado, a determinação do custo horário de equipamentos e a definição das produções das equipes mecânicas, pode-se avançar para o cálculo do custo unitário direto por serviço e total da obra, que corresponde ao produto dos custos unitários diretos pelas respectivas quantidades.

f) Cálculo da bonificação ou BDI (Benefícios e Despesas Indiretas)

Deve-se adotar o BDI calculado para o projeto em especifico, conforme metodologia apresentada no tópico 2.3.1 apresentado nesse trabalho. Após a conclusão do cálculo do BDI, este deve ser inserido na composição de custo unitário em forma de percentual.

g) Cálculo do custo unitário total ou do preço de venda do serviço

O preço de venda ou custo unitário total, é calculado em etapa posterior do orçamento e é definido como sendo a soma dos valores do custo direto do serviço e do BDI.

\section{METODOLOGIA}

Segundo Gil (2007), o entendimento sistemático correlacionado a resolução de problemas sugeridos, é denominado como pesquisa. Para elaboração deste trabalho, foram realizadas pesquisas de características: básica estratégica, exploratória, quantitativa e bibliográfica.

Metodologicamente, esta pesquisa é básica estratégica de natureza exploratória, devido ao fato de que foi desenvolvida em função de pressupostos e visa aprofundar o conhecimento acerca do processo de formação de composições de custos conforme apresentado. 
Referente ao tipo de abordagem do problema, trata-se de uma pesquisa de caráter quantitativa, visto que há partes objetivas que mensuram dados quantificados. Em relação ao procedimento técnico, utilizou-se a pesquisa bibliográfica, pois o trabalho foi elaborado com base em livros, artigos científicos, dissertações e teses.

\section{RESULTADOS E DISCUSSÃO}

Para efeito de análise e discussão, será apresentado a seguir, como demonstração dos embasamentos supramencionados no referencial teórico, duas composições de custos de serviço unitário com a sua devida quantidade estipulada, no qual, será possível realizar um comparativo entre estas, sendo uma confeccionada de maneira coerente e outra desprezando tal condição. A título de preciosismo será adotado o serviço de concretagem de lajes multifamiliares com encargos sociais inclusos a mão de obra, e deverá constar como requisitos de um projeto hipotético a utilização de bomba lança e Slump=190.

Tabela 5 - Análise do serviço de concretagem de lajes em edificação multifamiliares com serviço de bombeamento e concreto usinado com Slump $=190$.

\begin{tabular}{|c|c|c|c|c|c|c|c|c|}
\hline TIPO & 90856 & $\begin{array}{l}\text { CONCRETAGEM DE LAJES EM EDIFICAÇÕES } \\
\text { MULTIFAMILIARES FEITAS COM SISTEMA DE FÔRMAS } \\
\text { MANUSEÁVEIS COM CONCRETO USINADO } \\
\text { BOMBEÁVEL, FCK } 20 \text { MPA, LANÇADO COM BOMBA } \\
\text { LANÇA - LANÇAMIENTO, ADENSAMENTO E } \\
\text { ACABAMENTO. AF_06/2015 }\end{array}$ & M3 & COEF. & \multicolumn{2}{|c|}{$\begin{array}{l}\text { VALOR } \\
\text { UNITARIO }\end{array}$} & \multicolumn{2}{|c|}{$\begin{array}{l}\text { VALOR } \\
\text { TOTAL }\end{array}$} \\
\hline INSUMO & 39849 & $\begin{array}{l}\text { CONCRETO USINADO BOMBEAVEL, CLASSE DE } \\
\text { RESISTENCIA C20, COM BRITA 0 E 1, SLUMP }=190+/-20 \\
\text { MM, INCLUI SERVICO DE BOMBEAMENTO (NBR 8953) }\end{array}$ & M3 & 1,1100 & $\mathrm{R} \$$ & 315,96 & $\mathrm{R} \$$ & 350,72 \\
\hline COMP. & 88262 & $\begin{array}{llll}\text { CARPINTEIRO DE } & \text { FORMAS } & \text { COM } & \text { ENCARGOS } \\
\text { COMPLEMENTARES } & & & \end{array}$ & $\mathrm{H}$ & 0,1810 & $\mathrm{R} \$$ & 21,69 & $\mathrm{R} \$$ & 3,93 \\
\hline COMP. & 88309 & PEDREIRO COM ENCARGOS COMPLEMENTARES & $\mathrm{H}$ & 0,7250 & $\mathrm{R} \$$ & 21,81 & $\mathrm{R} \$$ & 15,81 \\
\hline COMP. & 88316 & SERVENTE COM ENCARGOS COMPLEMENTARES & $\mathrm{H}$ & 0,7220 & $\mathrm{R} \$$ & 15,04 & $\mathrm{R} \$$ & 10,86 \\
\hline COMP. & 90586 & $\begin{array}{l}\text { VIBRADOR DE IMERSÃO, DIÂMETRO DE PONTEIRA 45MM, } \\
\text { MOTOR ELÉTRICO TRIFÁSICO POTÊNCIA DE } 2 \text { CV - CHP } \\
\text { DIURNO. AF_06/2015 }\end{array}$ & $\mathrm{CHP}$ & 0,0670 & $\mathrm{R} \$$ & 1,13 & $\mathrm{R} \$$ & 0,08 \\
\hline COMP. & 90587 & $\begin{array}{l}\text { VIBRADOR DE IMERSÃO, DIÂMETRO DE PONTEIRA 45MM, } \\
\text { MOTOR ELÉTRICO TRIFÁSICO POTÊNCIA DE } 2 \text { CV - CHI } \\
\text { DIURNO. AF_06/2015 }\end{array}$ & $\mathrm{CHI}$ & 0,1140 & $\mathrm{R} \$$ & 0,19 & \multicolumn{2}{|l|}{$\mathrm{R} \$$} \\
\hline & & & \multicolumn{4}{|c|}{ TOTAL PARCIAL } & $\mathbf{R} \$$ & 381,41 \\
\hline & & & \multicolumn{2}{|c|}{ BDI } & \multicolumn{2}{|c|}{$25 \%$} & $\mathbf{R} \$$ & 95,35 \\
\hline & & & \multicolumn{4}{|c|}{ TOTAL PARCIAL } & $\mathbf{R} \$$ & 476,76 \\
\hline & & & \multicolumn{4}{|c|}{ QUANT. SERVIÇO } & \multicolumn{2}{|c|}{64,00} \\
\hline & & & \multicolumn{4}{|c|}{ PREÇO DE VENDA } & $\mathbf{R} \$$ & $0.512,80$ \\
\hline
\end{tabular}

Fonte: Criado pelo autor, com base no Catálogo de Composições, (SINAPI, 2017)

Pode-se observar que para o presente serviço foram adotados o concreto usinado com Slump $=190+/-20 \mathrm{~mm}$ e a utilização de bomba lança para distribuir o concreto. Essa 
composição está vinculada apenas ao serviço de concretagem, não sendo mensurado o posicionamento das malhas de aço ou confecção e posicionamento das formas. Atribui-se a quantidade de $64 \mathrm{~m}^{3}$ ao serviço em questão, sendo assim, o valor final do preço de venda totaliza-se em $\mathrm{R} \$ 30.512,80$ (trinta mil , quinhentos e doze reais e oitenta centavos).

Tabela 6 - Análise do serviço de concretagem de lajes em edificação multifamiliares sem o serviço de bombeamento e concreto usinado com Slump $=100$.

\begin{tabular}{|c|c|c|c|c|c|c|c|c|}
\hline TIPO & $\begin{array}{c}\text { COMP. } \\
01\end{array}$ & $\begin{array}{l}\text { CONCRETAGEM DE LAJES EM EIDIFICAÇÕES } \\
\text { MULTIFAMILIARES FEITAS COM SISTEM DE } \\
\text { FÔRMAS MANUSEÁ VEIS COM CONCRETO USINADO } \\
\text { BOMBEÁ VEL, FCK } 20 \text { MIPA - LANÇAMENTO, } \\
\text { ADENSAMENTO E ACABAMIENTO. AF_06/2015 }\end{array}$ & M3 & COEF. & \multicolumn{2}{|c|}{$\begin{array}{l}\text { VALOR } \\
\text { UNITARIO }\end{array}$} & \multicolumn{2}{|c|}{$\begin{array}{l}\text { VALOR } \\
\text { TOTAL }\end{array}$} \\
\hline INSUMO & 34492 & $\begin{array}{l}\text { CONCRETO USINADO BOMBEAVEL, CLASSE DE } \\
\text { RESISTENCIA C20, COM BRITA 0 E } 1 \text {, SLUMP }=100+/-20 \\
\text { MM, EXCLUI SERVICO DE BOMBEAMENTO (NBR } 8953)\end{array}$ & M3 & 1,1100 & $\mathrm{R} \$$ & 257,89 & $\mathrm{R} \$$ & 286,26 \\
\hline COMP. & 88262 & $\begin{array}{lrlll}\text { CARPINTEIRO DE } & \text { FORMAS } & \text { COM } & \text { ENCARGOS } \\
\text { COMPLEMENTARES }\end{array}$ & $\mathrm{H}$ & 0,1810 & $\mathrm{R} \$$ & 21,69 & $\mathrm{R} \$$ & 3,93 \\
\hline COMP. & 88309 & PEDREIRO COM ENCARGOS COMPLEMENTARES & $\mathrm{H}$ & 0,7250 & $\mathrm{R} \$$ & 21,81 & $\mathrm{R} \$$ & 15,81 \\
\hline COMP. & 88316 & SERVENTE COM ENCARGOS COMPLEMENTARES & $\mathrm{H}$ & 0,7220 & $\mathrm{R} \$$ & 15,04 & $\mathrm{R} \$$ & 10,86 \\
\hline COMP. & 90586 & $\begin{array}{l}\text { VIBRADOR DE IMERSÃO, DIÂMETRO DE PONTEIRA } \\
\text { 45MM, MOTOR ELÉTRICO TRIFÁSICO POTÊNCIA DE } 2 \\
\text { CV - CHP DIURNO. AF_06/2015 }\end{array}$ & $\mathrm{CHP}$ & 0,0670 & $\mathrm{R} \$$ & 1,13 & \multicolumn{2}{|l|}{$\mathrm{R} \$$} \\
\hline COMP. & 90587 & $\begin{array}{l}\text { VIBRADOR DE IMERSÃO, DIÂMETRO DE PONTEIRA } \\
\text { 45MM, MOTOR ELÉTRICO TRIFÁSICO POTÊNCIA DE } 2 \\
\text { CV - CHI DIURNO. AF_06/2015 }\end{array}$ & CHI & 0,1140 & $\mathrm{R} \$$ & 0,19 & \multicolumn{2}{|l|}{$\mathrm{R} \$$} \\
\hline & & & \multicolumn{4}{|c|}{ TOTAL PARCIAL } & $\mathbf{R} \$$ & 316,95 \\
\hline & & & \multicolumn{2}{|c|}{ BDI } & \multicolumn{2}{|c|}{$25 \%$} & $\mathbf{R} \mathbf{\$}$ & $\mathbf{7 9 , 2 4}$ \\
\hline & & & \multicolumn{4}{|c|}{ TOTAL PARCIAL } & $\mathbf{R} \mathbf{S}$ & 396,19 \\
\hline & & & \multicolumn{4}{|c|}{ QUANT. SERVIÇO } & \multicolumn{2}{|c|}{64,00} \\
\hline & & & \multicolumn{4}{|c|}{ PREÇO DE VENDA } & $\mathbf{R} \mathbf{\$}$ & $.356,18$ \\
\hline
\end{tabular}

Fonte: Criado pelo autor, com base no Catálogo de Composições, (SINAPI, 2017)

Mediante composição demonstrada acima, verifica-se um valor total de R $25.356,18$ (vinte cinco mil, trezentos e cinquenta e seis reais e dezoito centavos), sendo assim, pode-se verificar que esta representa uma falha pelo fato de não considerar a bomba lança para a distribuição do concreto, logo desencadeia uma característica diferente ao que seria recomendado pelo projeto hipotético, Slump $=100$, ocasionando uma diferença de $\mathrm{R} \$$ 5.156,62 (Cinco mil, cento e cinquenta e seis reais, e sessenta e dois centavos). Desta maneira, acarretaria em uma qualidade inferior ao esperado. Pode-se observar que se o volume do serviço de concretagem fosse maior o valor cresceria proporcionalmente, podendo chegar a valores consideravelmente altos.

Por termo, o resultado encontrado demonstra que a composição de custos realizada de forma correta e coerente aos projetos e todas as especificações técnicas em função da boa técnica construtiva, desencadeia segurança para a empresa, e/ou profissional liberal em 
relação a critérios de verificação de viabilidade de um empreendimento e execução de serviços dentro do valor determinado.

Deve-se atentar, também, aos valores de tributos no Brasil que são aplicados sobre a construção civil (DIAS, 2008). Pois, fazendo-se análise destes, conforme seção 2.2.3 (Tabela 4), observa-se que aproximadamente $41,1 \%$ do preço de venda dos serviços de engenharia são impostos. Logo, o restante $58,9 \%$ será o valor que o prestador de serviço utilizará para executar a obra e retirar respectivamente o seu lucro.

Discutindo a respeito de encargos sociais, é comum, contemporaneamente, ver casos de empregadores utilizarem mão de obra informal, infringindo assim as leis trabalhistas regidas pela CLT. Este fato ocorre comumente, em função da carga tributária incidente tanto nos custos, quanto nos preços de venda serem exorbitantes.

\section{CONSIDERAÇÕES FINAIS}

Esse trabalho teve o intuito de orientar e informar, aos estudantes e profissionais da área da construção civil, de forma sucinta e clara algumas metodologias, nomenclaturas e práticas para obtenção de uma composição de custo detalhada e coerente, visto a tamanha importância que esta ferramenta oferece para o planejamento e execução com excelência de serviços, permitindo-se a cobrança do valor denominado "justo/fidedigno" entre o prestador do serviço e o cliente.

Observa-se que quando as composições de custos são feitas sem os devidos fundamentos teóricos, orientações das entidades normativas e particularidades do projeto, pode-se ocorrer valores abaixo do necessário para execução do serviço, consequentemente, acarretará em atrasos no cronograma, estouro do orçamento, qualidade inferior ao desejado. Tratando-se de obras públicas, esse é um dos motivos que resultam no alto índice de sonegação de impostos e condições inapropriadas das obras.

Para o cálculo dos encargos sociais, cabe ao profissional da área de custos acompanhar a evolução das leis trabalhistas. Pois, estas são alteradas constantemente, e de acordo com a sua localidade, pode-se ocorrer variação de valores.

Portanto, para se atingir os objetivos impostos a cada obra a ser realizada, vislumbrando-se a excelência, deve-se entender que a gestão de custos, internalizada no segmento da engenharia civil, é uma ferramenta fundamental, pois, garante que o serviço seja concluído dentro do prazo estipulado (cronograma), com a qualidade esperada e pode gerar 
um fluxo de caixa positivo, que desencadeia automaticamente uma situação favorável no que se diz respeito ao ponto de equilíbrio. 


\section{REFERÊNCIAS}

GIL, A. C. Como elaborar projetos de pesquisa. 4. ed. São Paulo: Atlas, 2007.

GIL, ANTÔNIO CARLOS. Como Elaborar Projetos de Pesquisa. 5 Ed. São Paulo, Atlas, 2010 .

LAKATOS, E. MARIA; MARCONI, M. DE ANDRADE. Fundamentos de metodologia científica: Técnicas de pesquisa. 7 ed. - São Paulo: Atlas, 2010.

RENEKER, MAXINE H. A qualitative study of information seeking among members of na academic community: methodological issues and problems. Library Quarterly, v. 63, n. 4, Oct. 1993. p. 487-507.

PMI, Um Guia do Conhecimento em Gerenciamento de Projetos. Guia PMBOK®. Quinta Edição - EUA: Project Management Institute, 2013

SINAPI - Catálogo de Composições Analíticas. Disponível em 〈http://goo.gl/sinapi>. Acesso em 16 de Outubro de 2017.

SINAPI - Metodologias e Conceitos. Disponível em <http://goo.gl/sinapi $>$. Acesso em 16 de Outubro de 2017.

DIAS, Paulo R. V. Engenharia de Custos: Estimativa de Custos de Obras e Serviços de Engenharia. 2.ed. - Rio de Janeiro: IBEC, 2011.

DIAS, Paulo R. V. Engenharia de Custos: Uma Metodologia de Orçamentação para Obras Civis. 4.ed. - Rio de Janeiro: IBEC, 2011.

DIAS, Paulo R. V. Novo Conceito de BDI. 3.ed. - Rio de Janeiro: IBEC, 2008.

LIMMER, C.V. Planejamento, Orçamentação e Controle de Projetos e Obras. Rio de Janeiro: Livros Técnicos e Científicos Editora S.A., 1997.

MATTOS, Aldo Dórea. Como Preparar Orçamentos de Obras. 1.ed. - São Paulo: PINI, 2006. 
Anexo A - BDI

\begin{tabular}{|c|c|c|}
\hline \multicolumn{3}{|c|}{ PLANILHA DE CÁLCULO DO BDI } \\
\hline Valor do Contrato: & $\mathrm{R} \$$ & $150.000,00$ \\
\hline \multicolumn{3}{|l|}{ PARCELAS $(\%)$} \\
\hline Administração Central (AC) & $14,00 \%$ & \\
\hline \multirow{2}{*}{\multicolumn{3}{|c|}{$\begin{array}{l}\text { Custo Financeiro (CF) } \\
\qquad \mathrm{CF}=\llbracket\left(\left((1+\mathrm{t} / 100) \rrbracket^{\wedge}(\mathrm{n} / 30)-1\right) * 100\right.\end{array}$}} \\
\hline & & \\
\hline $\mathrm{t}=\%$ mês & & $1,00 \%$ \\
\hline $\mathrm{n}=\operatorname{dias}$ & & 45,00 \\
\hline Seguros $(\mathrm{S})$ & $1,00 \%$ & \\
\hline Garantias $(\mathrm{G})$ & $1,00 \%$ & \\
\hline Margem de Incerteza (MI) & $7,00 \%$ & \\
\hline Tributos sobre Receita $(\mathrm{T})$ & $6,65 \%$ & \\
\hline ISS & & $3,00 \%$ \\
\hline COFINS & & $3,00 \%$ \\
\hline PIS & & $0,65 \%$ \\
\hline Tributos sobre o Lucro & $2,28 \%$ & \\
\hline IRPJ (Lucro Presumido) & & $1,20 \%$ \\
\hline CSLL & & $1,08 \%$ \\
\hline Margem Líquida de Contribuição (MLC) & $11,72 \%$ & \\
\hline Margem Bruta de Contribuição (MBC) & $14,00 \%$ & \\
\hline BDI Contratantes $(\%)$ & & \\
\hline \multicolumn{3}{|c|}{$\mathrm{BDI}(\%)=(((1+\mathrm{AC}+\mathrm{CF}+\mathrm{S}+\mathrm{G}+\mathrm{MI}) /(1-(\mathrm{T}+\mathrm{MBC})))-1)$} \\
\hline BDI Prestador de Serviço (\%) & \multicolumn{2}{|c|}{$48,08 \%$} \\
\hline $\mathrm{BDI}(\%)=(((1+\mathrm{AC}+\mathrm{CF}+\mathrm{S}+\mathrm{G}) /$ & $(1-(\mathrm{T}+\mathrm{M}$ & C)) -1$)$ \\
\hline
\end{tabular}

Fonte: Novo Conceito de BDI (DIAS, 2008) 
Anexo B - ENCARGOS SOCIAIS

\begin{tabular}{|c|c|c|}
\hline \multicolumn{3}{|c|}{ COMPOSIÇÃO DE ENCARGOS SOCIAIS E TRABALHISTAS } \\
\hline Descrição & Horista \% & Mensalista \% \\
\hline \multicolumn{3}{|l|}{ GRUPO A } \\
\hline A1 - Previdência Social (INSS) & $20,00 \%$ & $20,00 \%$ \\
\hline A2 - Fundo de Garantia por Tempo de Serviço (FGTS) & $8,00 \%$ & $8,00 \%$ \\
\hline A3 - Salário educação & $2,50 \%$ & $2,50 \%$ \\
\hline A4 - Serviço Social da Indústria (SESI-SESC) & $0,00 \%$ & $1,50 \%$ \\
\hline A5 - Serviço Social de Aprendizagem Industrial (SENAI) & $0,00 \%$ & $1,00 \%$ \\
\hline A6 - Serviço de Apoio a Pequena e Média Empresa (SEBRAE) & $0,00 \%$ & $0,60 \%$ \\
\hline A7 - Instituto Nacional de Colonização e Reforma Agrária (INCRA) & $0,00 \%$ & $0,20 \%$ \\
\hline A8 - Seguro de Acidente de Trabalho & $3,00 \%$ & $3,00 \%$ \\
\hline TOTAL A & $33,50 \%$ & $36,80 \%$ \\
\hline \multicolumn{3}{|l|}{ GRUPO B } \\
\hline B1 - Repouso semanal remunerado & $17,99 \%$ & $0,00 \%$ \\
\hline B2 - Auxílio enfermidade & $0,92 \%$ & $0,69 \%$ \\
\hline B3 - Licença Paternidade / Maternidade & $0,08 \%$ & $0,06 \%$ \\
\hline B4 - 13º Salário & $11,06 \%$ & $8,33 \%$ \\
\hline B5 - Falta Justificada & $0,73 \%$ & $0,56 \%$ \\
\hline B6 - Feriados & $3,97 \%$ & $0,00 \%$ \\
\hline B7 - Férias & $11,03 \%$ & $8,34 \%$ \\
\hline B8 - Acidente de Trabalho & $0,12 \%$ & $0,09 \%$ \\
\hline B9 - Aviso Prévio Indenizado & $7,20 \%$ & $5,44 \%$ \\
\hline B10 - Aviso Prévio Trabalhado & $0,17 \%$ & $0,13 \%$ \\
\hline B11 - Dias de Chuva & $2,07 \%$ & $0,00 \%$ \\
\hline TOTAL B & $55,34 \%$ & $23,64 \%$ \\
\hline \multicolumn{3}{|l|}{ GRUPO C } \\
\hline $\mathrm{C} 1$ - Multa rescisão contrato ( $50 \%$ saldo FGTS ) & $5,23 \%$ & $3,97 \%$ \\
\hline C2 - Indenização adicional (Férias) & $3,23 \%$ & $2,44 \%$ \\
\hline C3 - Aviso Prévio indenizado & $0,61 \%$ & $0,46 \%$ \\
\hline TOTAL C & $9,07 \%$ & $6,87 \%$ \\
\hline \multicolumn{3}{|l|}{ GRUPO D } \\
\hline D1 - Reincidência de A sobre B & $17,65 \%$ & $6,66 \%$ \\
\hline D2 - Reincidência de (A - A9) sobre B9 & $0,88 \%$ & $0,65 \%$ \\
\hline D4 - Aviso Prévio sobre FGTS & $0,64 \%$ & $0,48 \%$ \\
\hline $\begin{array}{r}\text { TOTAL D } \\
\end{array}$ & $19,17 \%$ & $7,79 \%$ \\
\hline \multicolumn{3}{|l|}{ GRUPO E } \\
\hline E1 - Alimentação & $20,54 \%$ & $20,54 \%$ \\
\hline E2 - Vale transporte & $5,41 \%$ & $5,41 \%$ \\
\hline E3 - Café da Manhã & $6,13 \%$ & $6,13 \%$ \\
\hline E4 - Cesta Básica & $0,00 \%$ & $10,42 \%$ \\
\hline E5 - Equipamento de Proteção Individual (EPI) & $11,32 \%$ & $11,32 \%$ \\
\hline E6 - Seguro de Vida em Grupo & $0,00 \%$ & $0,00 \%$ \\
\hline E7 - Plano de Saúde & $0,00 \%$ & $0,00 \%$ \\
\hline TOTAL E & $43,39 \%$ & $53,81 \%$ \\
\hline TOTAL ENCARGOS SOCIAIS & $160,46 \%$ & $128,91 \%$ \\
\hline
\end{tabular}

Fonte: Criado pelo autor, com base no SINAPI (2017) e nas obras de Mattos (2006) e Dias (2011). 


\section{Anexo C - COMPOSIÇÃO DE SERVIÇOS E PREÇO DE VENDA.}

\begin{tabular}{|c|c|c|c|c|c|c|c|}
\hline \multicolumn{4}{|c|}{ COMPOSIÇÃO DE SERVIÇOS } & \multicolumn{2}{|c|}{$\mathbf{B D I}=$} & \multicolumn{2}{|c|}{$24,00 \%$} \\
\hline \multirow{2}{*}{$\sum_{\text {空 }}^{\underline{E}}$} & \multirow{2}{*}{$\begin{array}{l}\text { DISCRIMINAÇÃO DE } \\
\text { SERVIÇOS }\end{array}$} & \multirow{2}{*}{ 站 } & \multirow{2}{*}{ 运 } & \multicolumn{2}{|c|}{ CUSTO } & \multicolumn{2}{|c|}{ PREÇO DE VENDA } \\
\hline & & & & UNIT. & SERVIÇO & UNIT. & SERVIÇO \\
\hline 1 & \multicolumn{7}{|c|}{ SERVIÇOS PRELIMINARES } \\
\hline 1.1 & $\begin{array}{l}\text { PLACA DE OBRA EM CHAPA DE } \\
\text { ACO GALVANIZADO }\end{array}$ & M2 & 5,00 & 270,35 & $1.351,75$ & 337,95 & $1.689,74$ \\
\hline 1.2 & 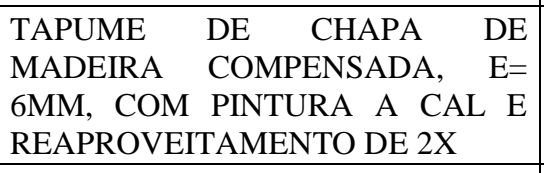 & M2 & 15,00 & 43,39 & 650,85 & 54,24 & 813,59 \\
\hline 1.3 & $\begin{array}{l}\text { DEMOLICAO DE ALVENARIA } \\
\text { ESTRUTURAL } \\
\text { VAZADOS DE CONCRETO }\end{array}$ & M3 & 24,00 & 43,11 & $1.034,64$ & 53,89 & $1.293,34$ \\
\hline 1.4 & $\begin{array}{lll}\text { DEMOLICAO } & \text { DE } & \text { CONCRETO } \\
\text { SIMPLES } & & \\
\end{array}$ & M3 & 3,00 & 163,81 & 491,43 & 204,77 & 614,31 \\
\hline 1.5 & \begin{tabular}{llr} 
DEMOLICAO & \multicolumn{2}{c}{ MANUAL DE } \\
ESTRUTURA & DE & CONCRETO \\
ARMADO & & \\
\end{tabular} & M3 & 2,50 & 163,81 & 409,53 & 204,77 & 511,92 \\
\hline & $\begin{array}{l}\text { SUB-TOTAL } \\
\end{array}$ & & & - & R\$ 3.938,20 & - & R\$ 4.922,91 \\
\hline
\end{tabular}

Fonte: Criado pelo autor, com base nas obras de Mattos (2006) e Dias (2011). 


\section{Anexo D - COMPOSIÇÃO DE CUSTO UNITÁRIO.}

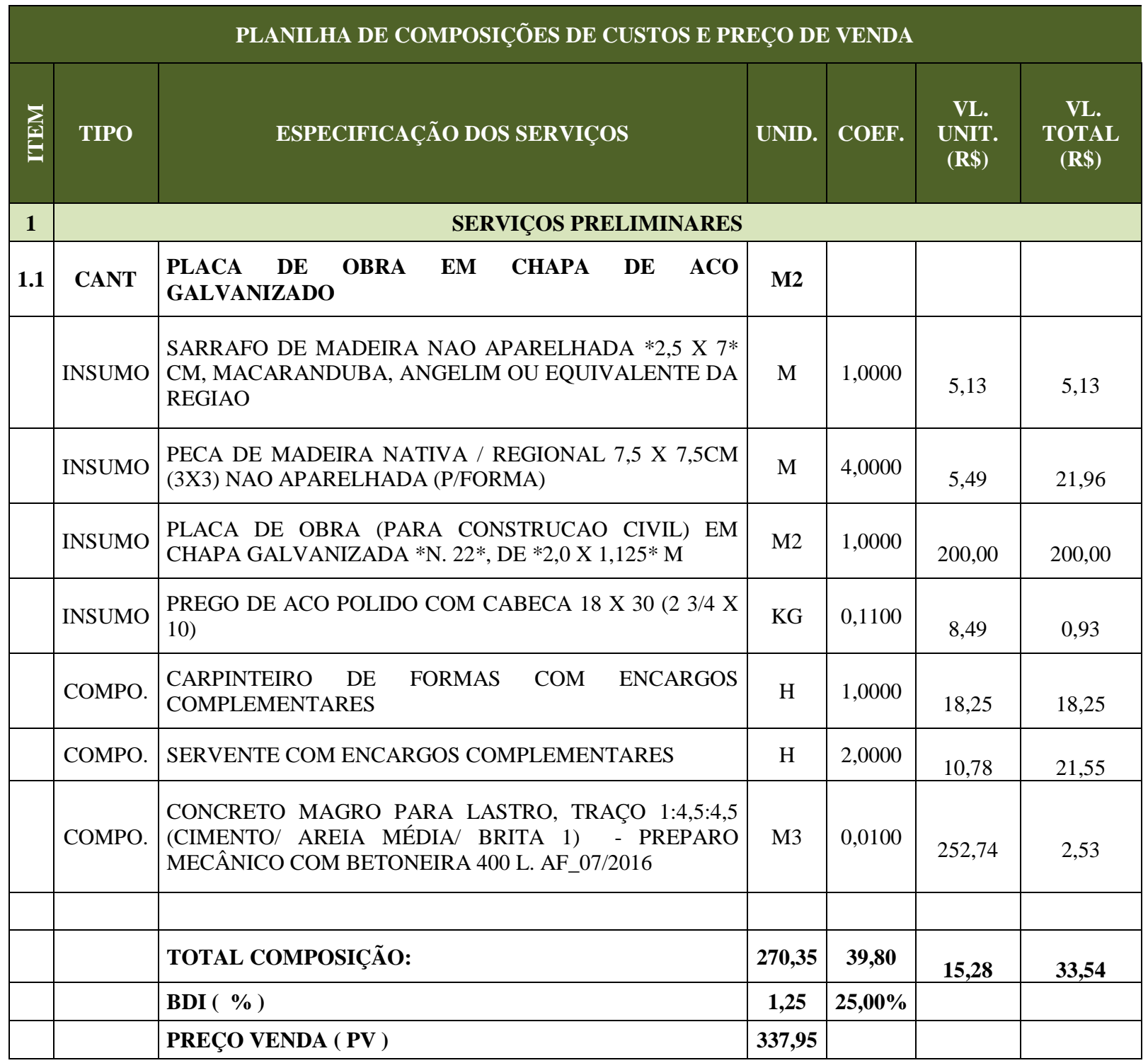

Fonte: Criado pelo autor, com base nas obras de Mattos (2006) e Dias (2011). 